\title{
Validation of a semi-quantitative $F F Q$ using food records as a reference in older women in the Kuopio Fracture Prevention Study (OSTPRE-FPS)
}

\author{
Arja T Erkkilä ${ }^{1, *}$, Ritva Järvinen ${ }^{2}$, Henna Karvonen ${ }^{2}$, Linda Keronen ${ }^{2}$ and \\ Marjo T Tuppurainen ${ }^{3}$ \\ ${ }^{1}$ Institute of Public Health and Clinical Nutrition, Public Health, University of Eastern Finland, PO Box 1627, \\ 70211 Kuopio, Finland: ${ }^{2}$ Institute of Public Health and Clinical Nutrition, Clinical Nutrition, University of Eastern \\ Finland, Kuopio, Finland: ${ }^{3}$ Department of Obstetrics and Gynecology, Kuopio University Hospital, and Bone and \\ Cartilage Research Unit, Clinical Research Center, University of Eastern Finland, Kuopio, Finland
}

Submitted 1 July 2011: Accepted 27 October 2011: First published online 29 November 2011

\begin{abstract}
Objective: To validate an eighty-nine-item semi-quantitative FFQ for measurement of nutrient intakes in elderly women.

Design: FFQ and $3 \mathrm{~d}$ food records were filled in by women participating in the Kuopio Fracture Prevention Study (OSTPRE-FPS). Data on intakes of energy, fat, protein, carbohydrate, fibre, $\mathrm{Ca}, \mathrm{Fe}, \mathrm{P}, \mathrm{K}, \mathrm{Mg}$, folic acid, vitamin $\mathrm{B}_{12}$, vitamin $\mathrm{C}$, vitamin $\mathrm{D}$ and vitamin $\mathrm{K}$ from ninety-nine women were available to assess the agreement of the two methods. Validity was assessed using correlation coefficients, cross-classification into quintile categories and Bland-Altman plots. Nutrients relevant to bone health were assessed.

Setting: OSTPRE-FPS in Finland.

Subjects: Elderly women with a mean age $71 \cdot 3$ years.

Results: The FFQ overestimated energy and nutrient intakes as compared with food records by 30-50\%. The highest correlation coefficients of the energyadjusted nutrient intakes between the methods were observed for fibre $(0 \cdot 60), \mathrm{Mg}$ $(0.56)$ and folic acid $(0 \cdot 49)$ and the lowest for protein and vitamin D (both $0 \cdot 19)$. The cross-classification of energy-adjusted nutrient intakes showed that on average $68 \%$ of the participants (range $62-78 \%$ ) were classified into the same or an adjacent quintile category.

Conclusions: The validity of energy and nutrient intakes measured with the FFQ was moderate as compared with $3 \mathrm{~d}$ food records in elderly women. The FFQ is a useful tool for the nutrient assessment of elderly women in epidemiological research.
\end{abstract}

Few studies have estimated the use and validity of FFQ in nutrient intake estimation in elderly populations ${ }^{(1-3)}$. However, determining nutrient intakes is critical in examining the effect of nutrition on health and disease. The FFQ is a useful tool to estimate long-term habitual dietary intake, can be self-administered and is relatively inexpensive. However, an FFQ should be tested in the specified population group before use ${ }^{(4)}$. Food records are a suitable reference method for assessing the validity of an FFQ because the errors associated with the two methods are independent ${ }^{(5)}$.

The present study examined the nutrient intakes measured in the Kuopio Fracture Prevention Study (OSTPREFPS). Therefore, especially the nutrients related to bone health were of interest in the validation. Our aim was to develop a semi-quantitative FFQ for elderly women and to evaluate the relative validity of nutrient intakes estimated by the FFQ as compared with $3 \mathrm{~d}$ food records.

\section{Experimental methods}

\section{Participants}

Participants were recruited from the OSTPRE-FPS ${ }^{(6)}$, which aims to examine the effect of $\mathrm{Ca}$ and vitamin $\mathrm{D}$ supplements on falls and fractures in women aged over 65 years during a 3-year intervention. The total number of women in the study was 3432, of whom 750 were randomly selected for a subsample participating in detailed examinations including measurement of bone mineral density and dietary records and FFQ. Dietary data used in the present study were collected at study year 3 (between April 2006 
and June 2006). The convenience sample in the validation study included 102 women. Three were excluded from the analyses due to high energy intake $>14 \cdot 7 \mathrm{MJ} / \mathrm{d}(3500 \mathrm{kcal} / \mathrm{d})$ in the FFQ, leaving ninety-nine women with food record and FFQ data.

The mean age of the women was 71.3 years in the validation study (SD $1 \cdot 7, \min 68 \cdot 5, \max 74 \cdot 3$ years). The mean height, weight and BMI of the women were 158 (sD 5) $\mathrm{cm}, 71 \cdot 2$ (sD 11.6) $\mathrm{kg}$ and $28.4(\mathrm{sD} 4 \cdot 8) \mathrm{kg} / \mathrm{m}^{2}$, respectively. The participants lived Kuopio Province including rural and urban areas.

The ethics committee of Kuopio University Hospital approved OSTPRE-FPS. The trial was registered at Clinicaltrials.gov under identifier NCT00592917 (registration date: 2 January 2008).

\section{Food records}

Current diet was assessed using $3 \mathrm{~d}$ food records including two consecutive weekdays and one weekend day. The instructions to complete a food record were mailed to the participants beforehand and they returned the records on a research visit at the Bone and Cartilage Research Unit (BCRU) at the University of Kuopio. Participants were instructed to write down everything they ate and drank and to evaluate the amount of food using household measures. The types of fats used on bread, in cooking and in baking were asked separately. In case of uncertainties in the food record, a nutritionist called the participant for more information.

Nutrient intakes were calculated using Diet32 software version 1.40 (Aivo Finland Oy, Turku, Finland). The Finnish food composition database, Fineli, was used in the calculations (National Institute for Health and Welfare, Helsinki, Finland).

\section{FFQ}

The FFQ was designed based on previous data on food consumption derived using food records in the same sample of Finnish elderly women at the baseline examination. The contributions of different food groups to energy, fat, vitamin and mineral intakes were evaluated and used to make the food list. Portion sizes for the FFQ were determined based on previous food records and an earlier Finnish $\mathrm{FFQ}^{(7)}$. Previous FFQ from the Finnish Health 2000 study $^{(7)}$, the British Aberdeen Prospective Osteoporosis Screening Study (APOSS) ${ }^{(8)}$ and the Swiss Evaluation of Nutrient Intake and Bone Ultra Sound (EVANIBUS) study ${ }^{(1)}$ were used in developing the questionnaire structures. The FFQ included fourteen pages and eighty-nine food items. The FFQ was designed to measure habitual food intake. The FFQ were given to the participants at the visit to the BCRU and they mailed the filled FFQ back using a prepaid envelope. Again, the nutritionist telephoned the participant if more information was needed.

The eighty-nine food items were classified as cereal products; dairy products; potato, rice and pasta; meat and poultry; fish and egg; food preparation and sauces; vegetables; fruit and berries; drinks; and sweets and snacks. All of the food groups contained an empty line for participants to add foods not listed. There were nine frequency options ranging from 'never or rarely' to 'over 5 times per day'. The type and amount of bread and fat spread were asked in more detail.

Daily nutrient intakes were calculated by multiplying the nutrient content per serving of each food item by the reported frequency of consumption and summing over all foods. Intake from supplements was not included in the calculated nutrient amounts. The nutrient contents were obtained from the Finnish food composition database, Fineli (National Institute for Health and Welfare). Nutrients calculated in the validation included protein, fat, carbohydrate, fibre, K, Ca, P, Mg, Fe, folic acid, and vitamins $\mathrm{B}_{12}$, $\mathrm{C}, \mathrm{D}$ and $\mathrm{K}$.

\section{Assessment of under-reporting at group level}

For evaluation of the plausibility of dietary intake data, the ratio of energy intake (EI) to estimated basal metabolic rate $\left(\mathrm{BMR}_{\mathrm{est}}\right)$ was calculated. BMR $\mathrm{est}$ was calculated based on body weight according to equations given by the Department of Health in the UK ${ }^{(9)}$. The EI:BMR ${ }_{\text {est }}$ cutoff value for under-reporting was chosen to be $1 \cdot 49$, as derived from Goldberg et al. ${ }^{(10)}$ and Black ${ }^{(11)}$.

\section{Statistical analyses}

All data analyses were performed using the SPSS statistical software package version $14 \cdot 0$ (SPSS Inc., Chicago, IL, USA). The nutrient intakes were adjusted for energy intake using the residual method ${ }^{(12)}$. Normality of the variables was tested by the Kolmogorov-Smirnov test. Pearson correlation coefficients were calculated to compare the two dietary assessments. In calculating the correlations logtransformed nutrient intakes were used to improve normality. The difference between the nutrient intakes from the two methods was tested using the paired-samples $t$ test. The women were classified into quintile categories of nutrient intakes and the percentage classified into the same, an adjacent or the opposite quintile category was calculated. Bland-Altman plots were used to determine if the difference in intake between the FFQ and food records varied depending upon the average nutrient intake ${ }^{(13)}$. The limits of agreement (mean difference $\pm 1.96 \mathrm{SD}$ of the difference between the methods) indicate whether the agreement is acceptable on an individual level.

\section{Results}

The mean nutrient intakes were higher when measured with the FFQ than with food records (Table 1). The least overestimation was observed for fat intake, $28 \%$, and most of the nutrients were overestimated by $30-50 \%$. Intakes of vitamins $\mathrm{C}$ and $\mathrm{K}$ were overestimated the most. The differences in the intakes of energy and energy-adjusted nutrients 
between the two methods were statistically significant for all of the nutrients (paired-samples $t$ test, $P<0 \cdot 05$ ).

Using the energy-unadjusted intakes, the lowest Pearson correlation coefficients were observed for protein $(r=0 \cdot 26)$ and vitamin $\mathrm{D}(r=0 \cdot 29)$, whereas the highest correlations were found for $\mathrm{Mg}(r=0.56)$ and fibre $(r=0.55$; Table 1$)$. Energy adjustment worsened the correlations for protein and vitamin D intakes ( $r=0 \cdot 19$ for both). The correlations improved for fibre, $\mathrm{P}$ and Ca with energy adjustment. When using the energy-adjusted nutrient intakes, the highest correlation coefficients were observed for fibre $(r=0 \cdot 60)$, $\operatorname{Mg}(r=0.56)$ and folic acid $(r=0.49)$.

The participants were divided into quintile categories of energy and energy-adjusted nutrient intakes as measured by the FFQ and food records to evaluate the ability of the methods to classify participants similarly. The average proportion of participants classified into the same quintile category was 31\% (Table 2). When the classification into the same or an adjacent quintile category was considered the average proportion of participants was $68 \%$. The highest proportion of women classified into the same or an adjacent quintile category was observed for fibre, $\mathrm{K}, \mathrm{Mg}, \mathrm{Fe}$ and folic acid intakes. The highest proportion of grossly misclassified participants was observed for vitamin D and protein intakes.

The Bland-Altman plots show the average and agreement limits of the difference in energy and energyadjusted nutrient intakes plotted against their mean (Fig. 1). For most of the nutrients the differences in intake were quite well within the $95 \%$ agreement limits. For energy and vitamin $\mathrm{D}$, the agreement was poorer when the average intake was higher.

\section{Discussion}

We evaluated the relative validity of energy and nutrient intakes estimated by a semi-quantitative FFQ as compared with a $3 \mathrm{~d}$ food record in free-living elderly Finnish women. We included especially nutrients relevant to bone health in the validation. We are not aware of any other FFQ specifically validated to be used in elderly subjects in Finland.

The FFQ overestimated energy and nutrient intakes as compared with the food records. Overestimation of energy and nutrient intakes is common when using FFQ and has been reported earlier in Finnish adult populations $^{(7,14)}$ and in a Dutch elderly population ${ }^{(3)}$. Two other studies in postmenopausal or elderly women have reported no significant differences in nutrient intakes measured with food records and $\mathrm{FFQ}^{(1,2)}$. It is also noteworthy that food records are prone to under-reporting, which was indicated by the mean ratio of EI:BMR est $_{\text {being }} 1 \cdot 23$ for food records, below the cut-off value $(1 \cdot 49)$. It has been suggested that a cut-off value of $1 \cdot 2$ is suitable for chair-bound or bed-bound individuals $^{(11)}$, which was barely met by the food record estimates. For the FFQ, the mean ratio of EI:BMR $\mathrm{est}(1 \cdot 60)$ 
was above the cut-off value. A slightly lower ratio $(1 \cdot 46)$ was reported earlier in 55- to 75-year-old women ${ }^{(3)}$ and even lower $(1 \cdot 24)$ in 75 - to 87-year-old women ${ }^{(1)}$.

The validation correlation coefficients are similar to those reported earlier in elderly ${ }^{(1,2,15)}$ and in Finnish studies $^{(7,14)}$. In agreement with our results the correlation coefficients have been low for protein ${ }^{(7,15)}$ and vitamin $\mathrm{D}^{(1)}$ in previous studies. Portion size estimation in food

Table 2 Classification of energy intake and energy-adjusted nutrient intakes into the same, adjacent or opposite quintile categories ( $n$ 99): elderly women (mean age 71.3 years) in the Kuopio Fracture Prevention Study (OSTPRE-FPS), Finland

\begin{tabular}{lccc}
\hline Nutrient & $\begin{array}{c}\text { Same quintile } \\
\text { category } \\
(\%)\end{array}$ & $\begin{array}{c}\text { Adjacent quintile } \\
\text { category } \\
(\%)\end{array}$ & $\begin{array}{c}\text { Opposite quintile } \\
\text { category } \\
(\%)\end{array}$ \\
\hline Energy & 23 & 45 & 0 \\
Protein & 29 & 30 & 6 \\
Fat & 27 & 41 & 5 \\
Carbohydrate & 33 & 34 & 4 \\
Fibre & 39 & 39 & 0 \\
K & 31 & 41 & 1 \\
Ca & 29 & 34 & 1 \\
P & 34 & 33 & 2 \\
Mg & 33 & 42 & 2 \\
Fe & 40 & 34 & 3 \\
Folic acid & 31 & 39 & 0 \\
Vitamin B & 26 & 37 & 4 \\
Vitamin C & 28 & 33 & 3 \\
Vitamin D & 34 & 30 & 8 \\
Vitamin K & 26 & 42 & 3 \\
\hline
\end{tabular}

records and FFQ is difficult for non-countable proteincontaining foods like meat and fish. This might have affected the low correlation of protein intakes even though its overestimation with the FFQ was similar to that of other nutrients. Fish, which is a major dietary source of vitamin $\mathrm{D}^{(16)}$, is consumed once or twice weekly. Thus the $3 \mathrm{~d}$ food record is not optimal for capturing the habitual fish intake and the validity is likely to be lower than for nutrients derived from multiple sources daily. The FFQ has been suggested to be a valid tool to estimate the intake of occasionally or episodically consumed foods ${ }^{(7)}$. According to the FFQ, almost half of the vitamin D came from fish and fish products $(4 \cdot 7 \mu \mathrm{g} / \mathrm{d})$.

The validity of measuring fibre intake was good as indicated by correlation coefficients and cross-classification. Similarly, earlier studies have reported good agreement between the two methods for assessment of fibre intake $^{(1,2,7,14,15)}$. Questions asking about the type and amount of bread were detailed, which could have contributed to the strong correlation of fibre intakes. Bread contributes a significant proportion of fibre intake in Finland (approximately $40 \%$ from bread in 65- to 74 -yearold women) ${ }^{(16)}$. Furthermore, significant proportions of $\mathrm{Mg}$ and $\mathrm{P}$ are derived from cereal products and the validity of their intake was good in the present study.

In estimation of diet and disease associations ranking of individuals according to their nutrient intakes is critical. We observed that ranking of individuals was adequate

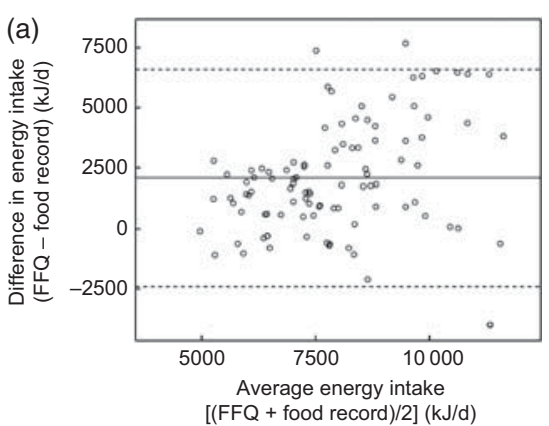

(d)

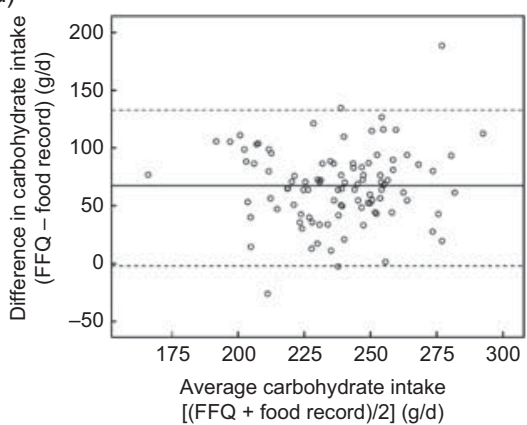

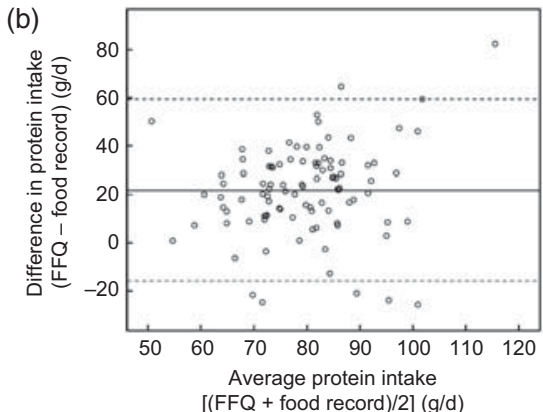

(e)

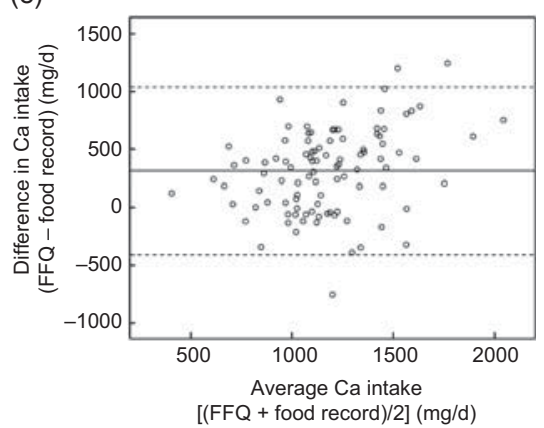

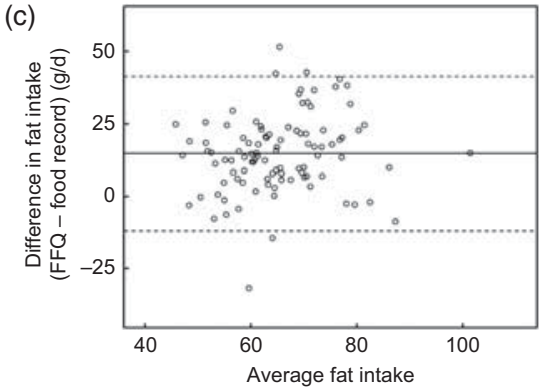

$[($ FFQ + food record $) / 2](g / d)$

(f)

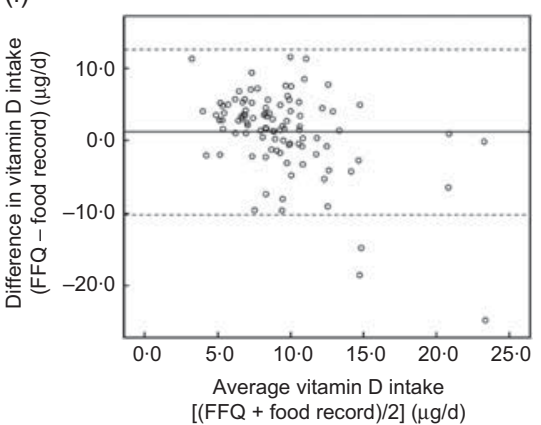

Fig. 1 Bland-Altman plots for intake of (a) energy, (b) protein, (c) fat, (d) carbohydrate, (e) calcium and (f) vitamin D measured with the FFQ and the $3 \mathrm{~d}$ food record, showing the difference in the energy or energy-adjusted nutrient intake between the methods plotted against the average energy or energy-adjusted nutrient intake based on both methods, among elderly women (mean age 71.3 years) in the Kuopio Fracture Prevention Study (OSTPRE-FPS), Finland. The mean $(-)$ and the limits of agreement (- - - -) for the differences are shown 
based on the cross-classification for the majority of the nutrients. Substantial gross misclassification occurred only for vitamin D and protein intakes. The poor classification of vitamin $\mathrm{D}$ and protein was also apparent by values not meeting the agreement limits in Bland-Altman plots. Of note is that also for vitamin $\mathrm{K}$ intake the ranking was valid, even though the absolute intakes poorly agreed. This has also been reported earlier ${ }^{(17)}$. Green salads, chicken and vegetable soups were the most important sources of vitamin $\mathrm{K}$ in the FFQ. It is likely that vegetable use is overestimated by the FFQ, and episodically consumed vegetables are poorly accounted for in food records ${ }^{(7,17)}$.

The number of women in the validation study can be considered reasonable ${ }^{(5)}$. Furthermore, many elderly women have regular dietary habits, which facilitates filling out an $\mathrm{FFQ}^{(7)}$. Portion sizes are typically smaller in elderly women than in adults. Portion sizes in our FFQ were smaller than the average adult portion sizes ${ }^{(18)}$. Nevertheless, we cannot exclude the possibility that the portion sizes were too big for the population and contributed to the overestimation of nutrient intakes. Furthermore, repeating the food records could have improved the agreement between the two methods. Food records and FFQ were collected in spring and early summer. Thus, we were not able to estimate the seasonal variation in dietary intake. Earlier it has been reported that consumption of leafy vegetables and vegetable fruits is higher in spring and summer in Finland ${ }^{(19)}$.

In conclusion, the FFQ overestimated absolute nutrient intakes. However, the FFQ is a valid tool for ranking individuals according to their nutrient intakes. The FFQ can be used to classify elderly women according to their nutrient intakes for research purposes in Finland.

\section{Acknowledgements}

The OSTPRE-FPS was supported by the Academy of Finland (M.T.T.), a Kuopio University Hospital EVO grant and by Leiras-Nycomed Ltd for the Ca and vitamin D supplementation. The authors do not have a conflict of interest. M.T.T. was responsible for the original OSTPRE-FPS design. A.T.E., R.J. and H.K. planned the validation study and the FFQ. L.K. performed the nutrient calculations. A.T.E. was responsible for the data analyses and writing the first draft. A.T.E., R.J. and L.K. contributed to the data interpretation. All authors contributed to revising the manuscript and approved the final version. The authors thank Susan LanhamNew for her advice in the FFQ development.

\section{References}

1. Dumartheray EW, Krieg M, Cornuz J et al. (2006) Validation and reproducibility of a semi-quantitative food frequency questionnaire for use in elderly Swiss women. J Hum Nutr Diet 19, 321-330.
2. Farrell VA, Harris M, Lohman TG et al. (2009) Comparison between dietary assessment methods for determining associations between nutrient intakes and bone mineral density in postmenopausal women. J Am Diet Assoc 109, 899-904.

3. Klipstein-Grobusch K, den Breeijen JH, Goldbohm RA et al. (1998) Dietary assessment in the elderly: validation of a semiquantitative food frequency questionnaire. Eur J Clin Nutr 52, 588-596.

4. Cade J, Thompson R, Burley V et al. (2002) Development, validation and utilisation of food-frequency questionnaires a review. Public Health Nutr 5, 567-587.

5. Willett W (1998) Nutritional Epidemiology, 2nd ed. New York: Oxford University Press.

6. Kärkkainen M, Tuppurainen M, Salovaara K et al. (2010) Effect of calcium and vitamin D supplementation on bone mineral density in women aged 65-71 years: a 3-year randomized population-based trial (OSTPRE-FPS). Osteoporosis Int 21, 2047-2055.

7. Paalanen L, Männistö S, Virtanen MJ et al. (2006) Validity of a food frequency questionnaire varied by age and body mass index. J Clin Epidemiol 59, 994-1001.

8. Macdonald HM, New SA, Fraser WD et al. (2005) Low dietary potassium intakes and high dietary estimates of net endogenous acid production are associated with low bone mineral density in premenopausal women and increased markers of bone resorption in postmenopausal women. Am J Clin Nutr 81, 923-933.

9. Department of Health (1991) Dietary Reference Values for Food Energy and Nutrients for the United Kingdom. London: HMSO.

10. Goldberg GR, Black AE, Jebb SA et al. (1991) Critical evaluation of energy-intake data using fundamental principles of energy physiology 1 . Derivation of cutoff limits to identify under-recording. Eur J Clin Nutr $\mathbf{4 5}$, 569-581.

11. Black AE (2000) Critical evaluation of energy intake using the Goldberg cut-off for energy intake:basal metabolic rate. A practical guide to its calculation, use and limitations. Int J Obes Relat Metab Disord 24, 1119-1130.

12. Willett W \& Stampfer MJ (1986) Total energy intake: implications for epidemiologic analyses. Am J Epidemiol 124, 17-27.

13. Bland J \& Altman D (1986) Statistical methods for assessing agreement between two methods of clinical measurement. Lancet 1, 307-310.

14. Erkkola M, Karppinen M, Javanainen J et al. (2001) Validity and reproducibility of a food frequency questionnaire for pregnant Finnish women. Am J Epidemiol 154, 466-476.

15. Smith W, Mitchell P, Reay EM et al. (1998) Validity and reproducibility of a self-administered food frequency questionnaire in older people. Aust $N Z J$ Public Health 22, 456-463.

16. Paturi M, Tapanainen H, Reinivuo H et al. (editors) (2008) Finravinto 2007 -tutkimus (The National FINDIET 2007 Study). Helsinki: National Public Health Institute.

17. Presse N, Shatenstein B, Kergoat M et al. (2009) Validation of a semi-quantitative food frequency questionnaire measuring dietary vitamin $\mathrm{K}$ intake in elderly people. J Am Diet Assoc 109, 1251-1255.

18. Sääksjärvi K \& Reinivuo H (2004) Ruokamittoja (Guide on Food Portions). Report B15/2004. Helsinki: National Public Health Institute.

19. Prasad M, Lumia M, Erkkola M et al. (2010) Diet composition of pregnant Finnish women: changes over time and across seasons. Public Health Nutr 13, 939-946. 